\title{
Numerical Study on the Effects of Anti-Snow Deflector on the Wind-Snow Flow Underneath a High-Speed Train
}

\author{
L. Cai, Z. Lou, T. Li and J. Zhang ${ }^{\dagger}$ \\ State Key Laboratory of Traction Power, Southwest Jiaotong University, Chengdu, Sichuan, 610031, China \\ †Corresponding Author Email: zhangjiye@my.swjtu.edu.cn
}

(Received January 21, 2020; accepted July 4, 2020)

\begin{abstract}
In order to study the effect of the anti-snow deflector on the wind-snow flow underneath a high-speed train, Detached Eddy Simulation (DES) approach and discrete phase model (DPM) are used to simulate the windsnow flow around the train. The distribution of snow particles underneath the train body is analyzed. Meanwhile, the influence of deflectors on the movement of snow particles around the train is investigated. The results show that lots of vortices shed from the bogie, and the entrainment vortices near the ground actuates the movement of the snow particles on the snow-covered track, which forms a wind-snow flow. The snow smoke around the train develops gradually from the bottom of the first bogie to the end of the tail car. The deflector installed in the front of the bogie will guide the vortices off the bogie region to the ground, which results in flying up more downstream snow particles and correspondingly the number of snow particles accumulated in the bottom of the rear car and around the rear skirt plate is increased. The installation position for the deflector has a certain effect on the snow accretion in the bogie region. When the deflector is installed in the front of the $2^{\text {nd }}$ and $4^{\text {th }}$ bogies, the snow particles captured in the bogie region are reduced by $42.3 \%$ and $15.6 \%$, respectively.
\end{abstract}

Keywords: High-speed train; Bogie; Underbody flow; Wind-snow flow; Discrete phase model; Deflector; Anti-snow.

\section{NOMENCLATURE}

$\begin{array}{ll}C_{d} & \text { particle resistance coefficient } \\ C_{p} & \text { pressure coefficient } \\ d_{p} & \text { particle diameter } \\ g & \text { inertia acceleration } \\ H & \text { train height } \\ L & \text { train length } \\ m_{p} & \text { particle mass } \\ P & \text { static pressure } \\ P_{\infty} & \text { reference pressure } \\ R e & \text { relative Reynolds number } \\ T_{r e f} & \text { reference time } \\ u & \text { air velocity }\end{array}$

\section{INTRODUCTION}

When a high-speed train (HST) runs on the snowcovered track, the train-induced slipstream makes the covered snow flying off the track, causing the

$\begin{array}{ll}u_{p} & \text { particle velocity } \\ u_{x} & \text { time-averaged streamwise velocity } \\ u_{y} & \text { time-averaged lateral velocity } \\ u_{z} & \text { time-averaged vertical velocity } \\ U & \text { normalized mean velocity } \\ U_{r e f} & \text { inlet velocity } \\ \mathrm{W} & \text { train width } \\ \mu & \text { aerodynamic fluid viscosity } \\ \rho & \text { air density } \\ \rho_{p} & \text { particle density } \\ \tau_{r} & \text { particle relaxation time }\end{array}$

snow accretion in the bogie region (Kamata and Yokokura, 2019). Due to the heat sources such as brake disc and traction motor in the bogie region (Milosevic et al. 2012; Petry et al. 2018; Nategh et al. 2019), some of the snow particles entering the 
bogie region will melt into water droplets, and therefore the ice is easily formed. The freezing snow can damage the flexible components of the bogie and also significantly affect the movement of the elastic suspension, thus affecting the running safety of trains (Kloow, 2011). To minimize the impact of snow accretion on the running safety of trains, the railway manufactory has developed a variety of methods to remove the snow and ice on the bogie.

The process of snow particles being carried into the bogie region by the slipstream is a phenomenon of aeolian transport. The method of reducing snow particles entering the bogie region can be studied in terms of flow control which slows down the snow accumulation speed in the bogie region. For instance, Shishido et al. (2009) investigated the influence of the side deflector of the train skirt plate on the snow accretion in the bogie region in a snowfall wind tunnel. studied the influence of the triangular prism spoiler on the snow accretion in the bogie region in a low-temperature wind tunnel. Gao et al. (2019) used discrete phase model (DPM) to study the effects of the deflector at the bottom of the end wall of the bogie cavity on reducing the snow accretion in the bogie region. The effects of deflector, shape of bogie cavity, anti-snow shields on reducing snow accretion of bogie and brake clamp were investigated using DPM (Wang et al. 2018a,b, 2019a,b). The previous studies show that installing a deflector under or on the side of the train or increasing the inclination of the end walls of the bogie cavity can effectively reduce the snow accumulation in the bogie region.

With the increasing availability and accuracy, computational fluid dynamics (CFD) is widely used in the prediction of snowdrift around buildings (Tominaga, 2018). The approaches include EulerEuler and Euler-Lagrangian methods. Discrete phase model (DPM) belongs to Euler-Lagrangian frames, which has been proved to be highly accurate in solving the gas-solid two-phase flow problems (Patankar and Joseph, 2001; Chiesa et al. 2005; Pankajakshan et al. 2011). Paradot et al. (2014) verified the snow accretion on a bogie calculated using DPM with the experimental data. And the snow accumulation process on the bogie was simulated using DPM together with mesh deformation tools. Gao et al. (2019) investigated the snow accretion on the bogie of a HST with DPM and tested the snow accretion on the bogie with sawdust substituting snow particles. The distribution of snow particles on the surface of bogie calculated by DPM was consistent to the experimental results. As the DPM contains all the information of particle motion, it is widely applied to study the snow accretion of bogies (Wang et al. 2018a,b, 2019a,b; Gao et al. 2019; Xie et al. 2017).

CFD has been also widely used in the research of the flow around trains or underbody flow of trains. Li et al. (2019) compared the different RANS turbulence model on the flow around a simplified train. It is found that the shear stress transport (SST) model has an excellent ability in the prediction of aerodynamic forces of the train. Soper et al. (2018) compared the performance of CFD and dynamic model wind tunnel test method for the flow beneath the train. Zhu and $\mathrm{Hu}$ (2017) studied the force characteristics of airflow beneath a HST bogie using Detached Eddy Simulation (DES) approach. Paz et al. $(2017,2019)$ used delayed DES (DDES) and dynamic mesh to investigate the effects of true shape track beds and ballasts on underbody flow as trains pass. DES approach has high accuracy in predicting the separation position and aerodynamics of the large separation flow of ground cars (Kapadia et al. 2003; Sreenivas et al. 2006; Guilmineau et al. 2011; Kang et al. 2012; Li et al. 2020). It is proved that DES approach has the ability to accurately predict in the slipstream, wake and underbody flow of HST (Zhang et al. 2016; Wang et al. 2017,2018; Xia et al. 2017). Meanwhile, it also performs well in solving flows around aircraft landing gear (Hedges et al. 2002, Xiao et al. 2013, Dong et al. 2018) and bogie (Sima et al. 2008; Wang et al. 2019a). DES approach has a strong dependence on the computational mesh. The inappropriate mesh will start the sub-mesh model in advance, which will lead to the stress loss of boundary layer and mesh-induced separation (Vatsa and Lockard, 2010). The Improved Delayed DES (IDDES) model reduces the dependence on mesh and makes DES class approach more suitable for engineering field. In this paper, IDDES and DPM are selected to study the influence of anti-snow deflector on wind-snow flow beneath HST.

This paper mainly studies the influence of the antisnow deflector on wind-snow flow underneath the train. The content of this paper is as follows. In section 2, the train model, computational domain and boundary conditions, snow injector settings and calculation mesh are described in section 2. And in the next section, the characteristics of snow around the train and the influence of anti-snow deflector of bogie on snow particle transport beneath the train are analyzed and discussed. Finally, the conclusion is described in section 4 .

\section{NUMERICAL SETUPS}

\subsection{Geometric Model}

The numerical model consists of 3 cars of $\mathrm{CRH} 3$ high-speed train, including one head car, one intermediate car, one tail car and six simple bogies. For simplicity, the $1^{\text {st }}$ bogie is named as bogie 1 , and the same rules for the remaining bogies. The length, width and height of the train are $\mathrm{L}=76.5 \mathrm{~m}$, $\mathrm{W}=3.3 \mathrm{~m}$ and $\mathrm{H}=3.9 \mathrm{~m}$, respectively. Three different models (Case 1, Case 2 and Case 3 ) are established to analyze the influence of anti-snow deflector on the wind-snow flow. Case 1 does not contain any deflector. Case 2 installed a deflector in the front of the $2^{\text {nd }}$ bogie, and Case 3 in the front of the $4^{\text {th }}$ bogie. The installation position and size of the deflector are shown in Fig. 1. To compare with the numerical results of the vertical arrangement of the snow ejector in front of the car, the shape and installation position of the deflector in this study is the same as the one used by Gao et al. (2019). 

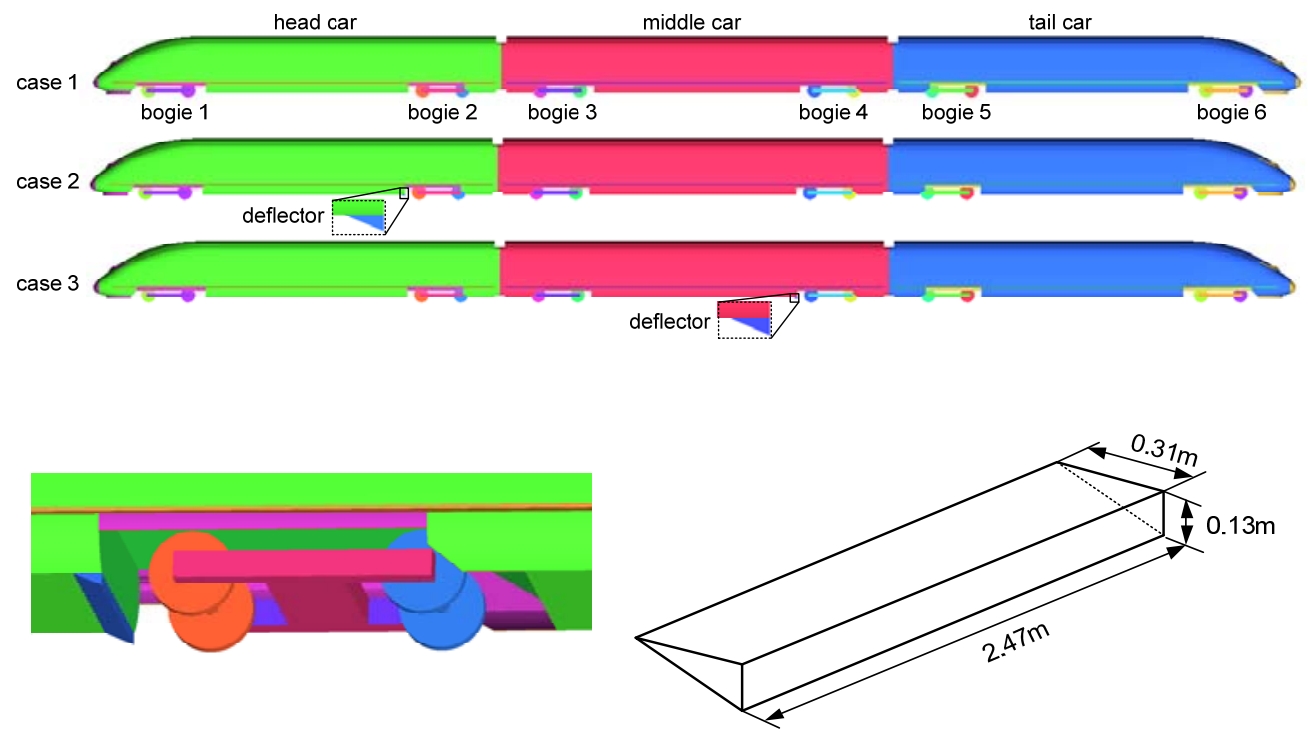

Fig. 1. Train model and anti-snow deflector.

All simulations presented are completed in the commercial code Fluent 15.0. The computational domain is shown in Fig. 2. Its length, width and height are $216.5 \mathrm{~m}, 60 \mathrm{~m}$ and $100 \mathrm{~m}$, respectively. The inlet is $40 \mathrm{~m}$ away from the nose tip of the head car. The outlet is $100 \mathrm{~m}$ away from the nose tip of the tail car. The bottom of the train is $0.35 \mathrm{~m}$ away from the ground. In the numerical simulation, the inlet is set as velocity inlet. The outlet is set as a pressure outlet. The ground is set as a sliding wall. The side and top surfaces are set as symmetrical wall. The wheels are set as rotating wall. The specific boundary conditions are shown in Table 1.

To investigate the characteristics of the snow particles on the walls, the bogie and the car body use a user-defined function (UDF) in discrete phase boundary conditions. The UDF is used to count the total number of impact snow particles on the wall. The statistical results are stored in the user-defined memory (UDM). A predefined macros DEFINE_DPM_BC provided by Fluent is used to define the UDF to specify user-defined boundary conditions for particles. The function is executed every time a particle touches a boundary of the domain. When the particle hits the wall, the value of the UDM that stores the number of particle impacts will be increased by 1 , and then the state of the particle will be marked as PATH_END. For the return status PATH_END, the particle will be assumed to escape from the domain.

To save computing resources, a transverse sampling plane is set at the rear of the train in the computational domain (see Fig. 2). With the custom sampling function (UDF), snow particles passing the sampling surface will be released from the computational domain and will not be considered in the subsequent calculation. Therefore, the number of tracking snow particles can be decreased substantially. In all, the number of particles tracked in this study is about 26 million for a stable windsnow flow.

\subsection{Methodology for CFD Analysis}

In this paper, an IDDES approach (Shur et al. 2008) based on the SST $k-\omega$ model is used to capture more vortices in the wake flow between the train and track bed. As a RANS-LES hybrid technique, IDDES approach combines the advantages of the delayed eddy simulation (DDES) and the wallmodelled large eddy simulation (WMLES). The DDES approach provides the delayed option to protect the boundary layer from the limiter. The complete formulations for the IDDES can be referred by Shur et al.(2008) and Gritskevich et al.(2012)

The trajectory of snow particles predict by the DPM. The snow particles are assumed to be spheres without considering the impact of the collision between them. The force balance equation of snow particles is as follows:

$m_{p} \frac{d u_{p}}{d t}=m_{p} \frac{u-u_{p}}{\tau_{r}}+m_{p} \frac{g\left(\rho_{p}-\rho\right)}{\rho_{p}}$

Where $m_{p}$ is the particle mass; $u$ is air velocity; $u_{p}$ is snow particle velocity; $\rho$ is air density, and $\rho=1.225 \mathrm{~kg} / \mathrm{m}^{3} ; \quad \rho_{p}$ is snow particle density; $\tau_{r}$ is particle relaxation time calculated by:

$\tau_{r}=\frac{\rho_{p} d_{p}^{2}}{18 \mu} \frac{24}{C_{d} R e}$

Here, $\mu$ is aerodynamic fluid viscosity, $d_{p}$ is the diameter of snow particle, $C_{d}$ is the resistance coefficient of particle, Re is the Reynolds number, 


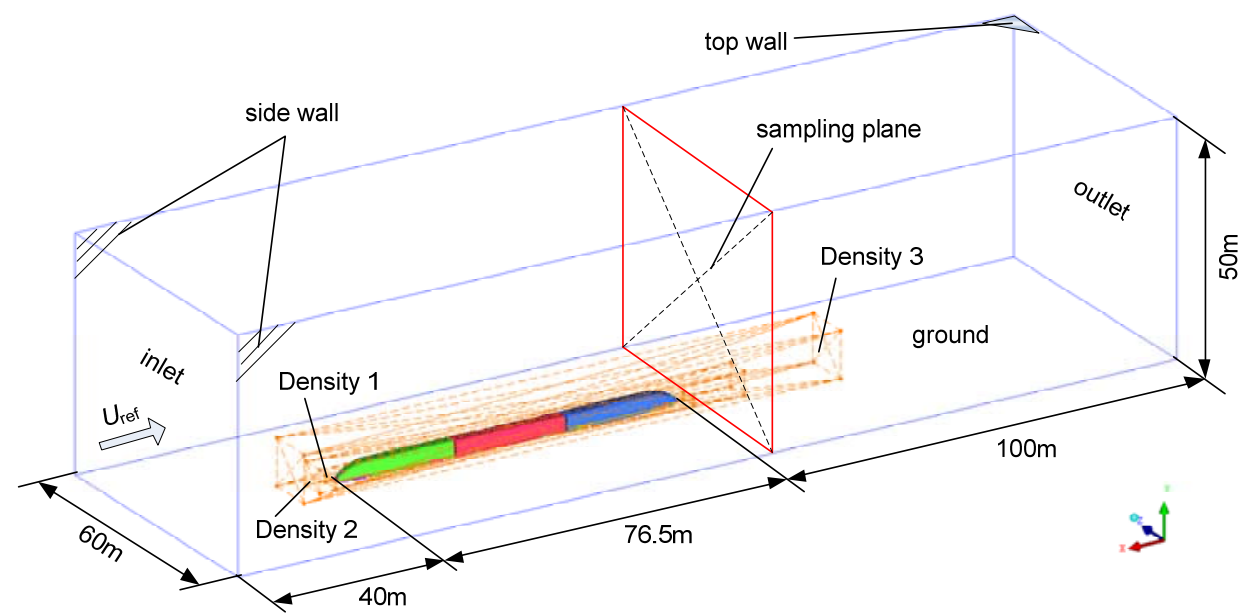

Fig. 2. Computational domain.

which is defined as

$\operatorname{Re} \equiv \frac{\rho d_{p}\left|\vec{u}_{p}-\vec{u}\right|}{\mu}$

In the previous simulations of the wind-snow (Wang et al. 2018a,b, 2019a,b; Gao et al. 2019), snow particles injected into the train underbody along the flow direction were arranged by a transverse jet plane. In this way, the snow particles cover the entire gap between the bottom of car and the rail. The snow particles injected in the front of the car gradually reach the downstream bogies region over time. Due to the capture of the upstream walls of the train, the number of snow particles entering the downstream region beneath the car will decrease progressively. This method ignores the vertical development of wind-snow flow beneath HST and is not suitable for the underdeveloped situation. Observing the distribution of wind-snow flow caused by the running train in actual (Kloow, 2011), we can see that the snow smoke develops from the region beneath of the head car. Its coverage becomes higher gradually, and finally forms the snow smoke that surrounds the whole train.

Different from the layout of the snow injector in the literature (Wang et al. 2018a,b, 2019a,b; Gao et al. 2019), we adopts the horizontal layout of the snow injector on the ground beneath the train. The particles are emitted downstream in the horizontal direction. The arrangement of the injector is shown in Fig. 3. The horizontal inject plane is $5 \mathrm{~cm}$ above the ground, the injection points are arranged at an interval of $0.1 \mathrm{~m}(760 \times 40=30400$ points $)$. The train speed is $70 \mathrm{~m} / \mathrm{s}$. At the injection point, a particle with an initial velocity $(-70,0,0) \mathrm{m} / \mathrm{s}$ was launched in the streamwise direction every $0.0005 \mathrm{~s}$, the diameter is $0.2 \mathrm{~mm}$ and density is $150 \mathrm{~kg} / \mathrm{m}^{3}$. The mass flow rate of the snow particles emitted from the injector was $0.0382 \mathrm{~kg} / \mathrm{s}$.

The stochastic tracking approach was adopted for DPM. The random walk model (RWM) (Gosman and Loannides, 1983) was selected to simulate the influence of turbulence on the diffusion of snow particles. In the stochastic tracking approach, it predicts the turbulent dispersion of particles by integrating the trajectory equations for individual particles using the instantaneous fluid velocity, $\bar{u}+u^{\prime}(t)$, along the particle path during the integration. Fluent uses a stochastic method to determine the instantaneous gas velocity. Firstly, the steady flow field is solved, and then the transient calculation is started with the steady flow field. The DPM calculation is started when the transient flow time reaches $1.0 \mathrm{~s}$. The time step of $\Delta t_{\text {IDDES }} \approx 0.0025 T_{\text {ref }}\left(T_{\text {ref }}=H / U_{\text {ref }}\right)$ is required due to the restrictions of the Courant number (Wang, et al. 2017). In this paper, the time step for each case is fixed with $1 \times 10^{-4} \mathrm{~s}$, and the CFL number of most cells is less than 1. Each time step iterates 20 times, and the total solution time of $\mathrm{DPM}$ is $2.5 \mathrm{~s}$.

Table 1 Boundary conditions

\begin{tabular}{|c|c|c|}
\hline boundary & $\begin{array}{c}\text { boundary } \\
\text { condition type }\end{array}$ & parameter \\
\hline inlet & Velocity inlet & $\begin{array}{c}\mathrm{X}=-70 \mathrm{~m} / \mathrm{s}, \text { Escape } \\
\text { Turbulence Intensity: } 1 \% \\
\text { Turbulence Viscosity } \\
\text { Ratio: } 10 \%\end{array}$ \\
\hline outlet & Pressure outlet & $0 \mathrm{~Pa}$, Escape \\
\hline $\begin{array}{c}\text { side and } \\
\text { top wall }\end{array}$ & Symmetry & - \\
\hline ground & $\begin{array}{c}\text { Moving wall: } \\
\text { transition }\end{array}$ & $\mathrm{X}=-70 \mathrm{~m} / \mathrm{s}$, Escape \\
\hline wheel & $\begin{array}{c}\text { Moving wall: } \\
\text { rotation }\end{array}$ & $152 \mathrm{rad} / \mathrm{s}$, UDF \\
\hline bogie & No-slip wall & UDF \\
\hline train body & No-slip wall & UDF \\
\hline
\end{tabular}

The finite volume method is used to discretize the governing equations. The equations (the instantaneous continuity and momentum equations, 


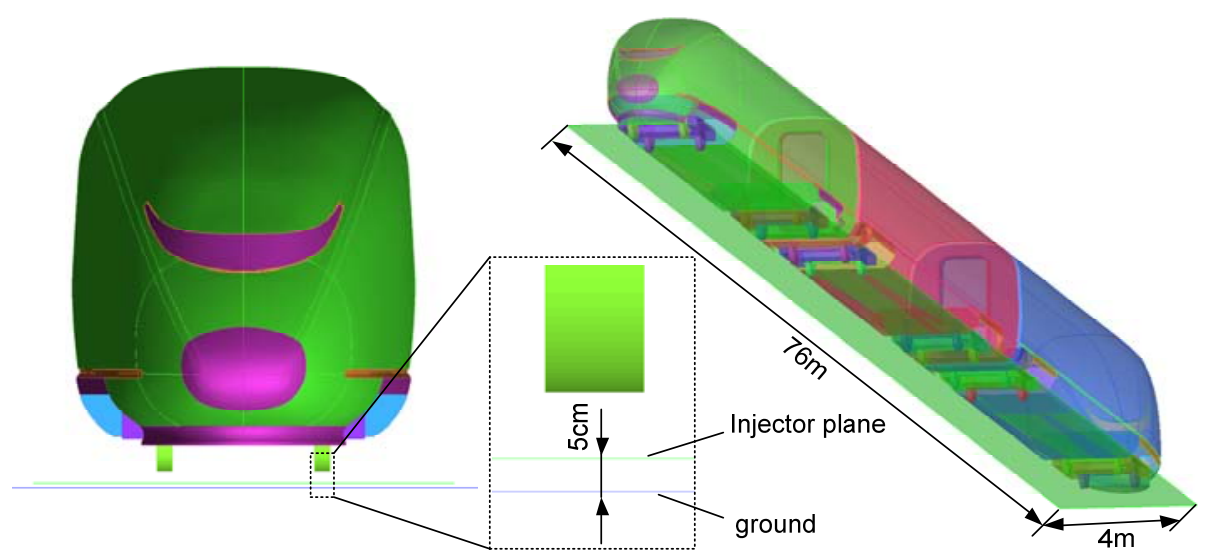

Fig. 3. Location and size of snow injector plane below the train.

The turbulence kinetic energy, $k$, and the specific dissipation rate, $\omega$, transport equations) is solved by SIMPLE algorithm. Bounded central differencing scheme is adopted for the momentum term. The pressure terms, turbulent flow energy and turbulent diffusivity is discrete using second order upwind scheme. And the time term is discrete using bounded second order implicit scheme.

\subsection{Computational Mesh and Validation}

Two sets of tetrahedral grids named as medium and fine mesh are generated. The medium and fine meshes have 57 and 98 million volume cells, respectively. Figure 5 shows the comparison in the pressure coefficient $C_{p}$ for numerical and the experimental results (Xia et al. 2017) at the centerline of the upper surface of the head and tail cars. The static pressure on the train and mean velocity component in the travelling direction are normalized as follows:

$$
\begin{aligned}
& C_{p}=\left(P-P_{\infty}\right) / 0.5 \rho U_{r e f}^{2} \\
& U=\sqrt{\left(U_{r e f}-u_{x}\right)^{2}+u_{y}{ }^{2}+u_{z}{ }^{2}} / U_{r e f}
\end{aligned}
$$

Where $P$ is the static pressure, $P_{\infty}$ is the reference pressure, $U_{r e f}$ is the inlet velocity, $u_{x}, u_{y}$ and $u_{z}$ are the streamwise, the lateral and the vertical components of air velocity.

The time-averaged pressure coefficients $C_{p}$ obtained using two different grids match the experimental values (Xia et al. 2017). Therefore, the medium mesh is chosen to simulate the windsnow flow around the train. The details of the medium mesh in this study are shown in Fig. 4. Two mesh refinement regions are added around the train, and a local mesh refinement region is set at the bottom of the train. The near-wall region is divided into 15 layers of prism mesh The thickness of the first layer is $0.5 \mathrm{~mm}$, which is the same as that in the literature (Paz et al. 2019). In this paper, $\mathrm{y}^{+}$is mainly in the range of 5 and 60. IDDES uses
LES method in the vortex core region far away from the wall. If all scales eddies are to be calculated, the existing computing capacity cannot meet the requirements of mesh quantity. Therefore, only large-scale eddies played a significant role in aerodynamic forces and particle diffusion are considered in this paper by using the medium mesh. The maximum mesh size of the region beneath the train for the medium mesh is $32 \mathrm{~mm}$.

Figure 6 shows the comparison in the normalized mean velocity component $U$ using the numerical simulation, the full-scale test (Jönsson et al. 2014) and the scale model test (Deeg et al. 2008). Due to the difference in the length of the adopted train, the horizontal coordinate data is aligned for comparison. The full scale measurements was conducted for an Italian high-speed train ETR500 (Jönsson et al. 2014), and the scale model test was measured on the ICE3 (Deeg et al. 2008). The numerical results accurately capture the peak velocity fluctuation when the head and tail cars pass by. The velocity around the head and tail carsis close to the test results, and the velocity variation trend matches the measured data for ETR500. However, it should be noted that the underbody flow is quite sensitive to the geometry (Deeg et al. 2008; Jönsson et al. 2014; Wang et al. 2018; Zhang et al. 2018). The train model adopted in this paper is not exactly the same as that in the experiments. Therefore, the calculated result is different from the experimental data in certain. The comparison between the calculated results and the experimental results shows that the numerical method and the medium mesh used in this paper are reasonable.

\section{Results AND Discussion}

\subsection{Snow Smoke Around the Train}

Figure 7 shows the distribution of snow particles around the train at different DPM simulation time $t_{\mathrm{p}}$. The snow particles are colored by the dimensionless time $t_{\mathrm{pr}} / t_{\mathrm{p}}\left(t_{\mathrm{pr}}\right.$ is the particle residence time). At first, the snow particles on the ground 


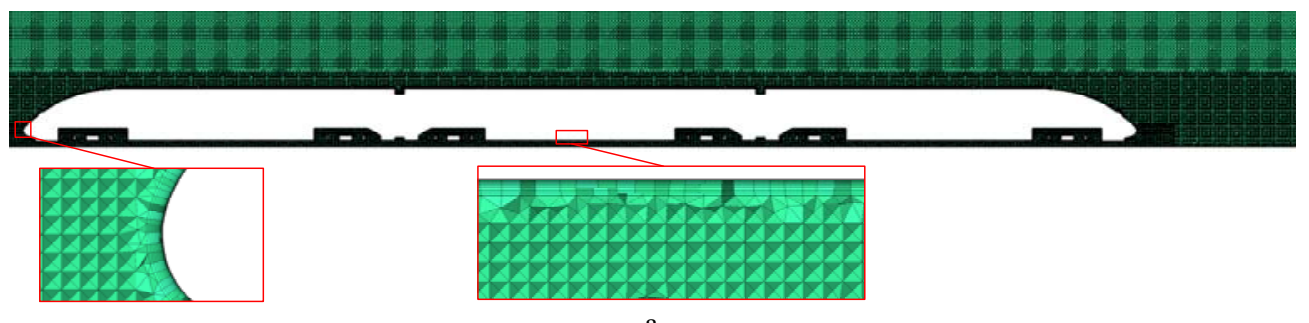

a

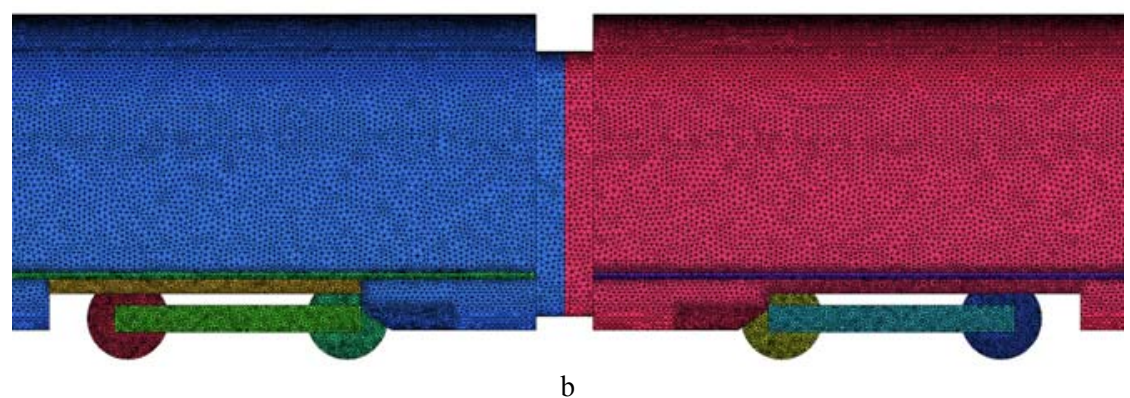

Fig. 4. Computational mesh: (a) volume mesh; (b) surface mesh.

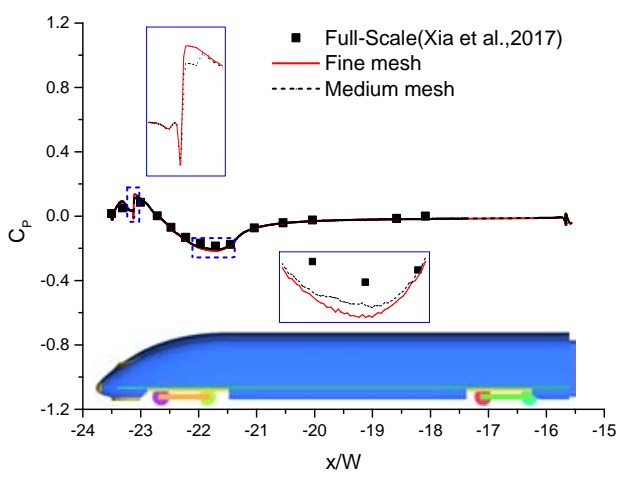

a

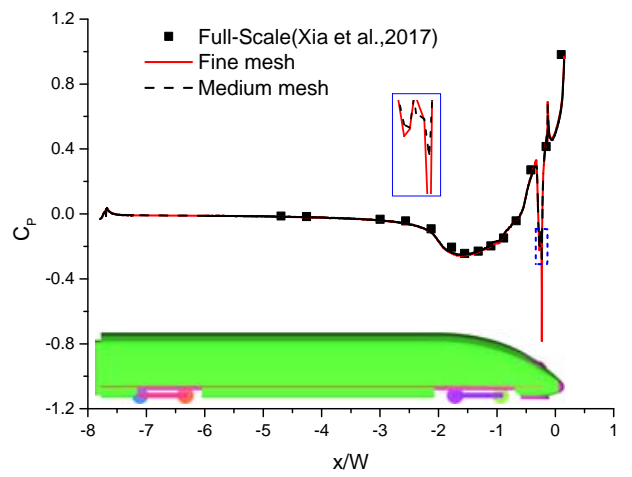

b

Fig. 5. Comparison in pressure coefficients at the center line of the upper surface of the car body between numerical simulation and experiments: (a) tail car; (b) head car.

gradually move to the upper region around the train. After $0.1 \mathrm{~s}$, the particles reach the bottom surface of the train, and $0.5 \mathrm{~s}$ later, the particles reach the height of the train. The particle residence time $t_{\mathrm{pr}}$ of most snow particles around the train is less than $t_{\mathrm{p}}$. It can be seen that the wind-snow flow around the train has reached the relatively stable state after $t_{\mathrm{p}}$ $=1.5 \mathrm{~s}$. At that state, the distribution height of snow particles around the train gradually increases from the head car to the tail car, reaching the same height of the train at the tail car. In particular, as the snow particles rolled up by the underbody airflow are too late to enter the bogie 1 region, the snow particles are less accumulated in the region of the bogie 1. The distribution of snow smoke caused by trains in an actual operation was reported by Paradot et al. (2014). The distribution trend of snow particles along the longitudinal direction of the train using numerical simulation is consistent with the actual situation.

In order to compare the distribution of snow concentration along the longitudinal direction beneath the train, three longitudinal monitoring lines parallel to the $\mathrm{X}$ axis are set up and shown in Fig. 8. The lines 1 and 2 are located beneath the car and line 3 is located on the side of the skirt plate. The average volume fraction of snow particles on the monitoring lines are calculated (see Fig. 9). It shows that the snow particle concentration under the train (line 1 and 2) begins to increase rapidly at the rear of the bogie 1 and reaches the average level at the middle of the head car. The peak value of snow concentration underneath the train appears below the bogie, and the snow concentration below the bogie 3 is the highest. The snow concentration on the skirt plate side (line 3) increase gradually from the rear of the bogie 1 and reached the average level at the central of the middle car. It shows that 


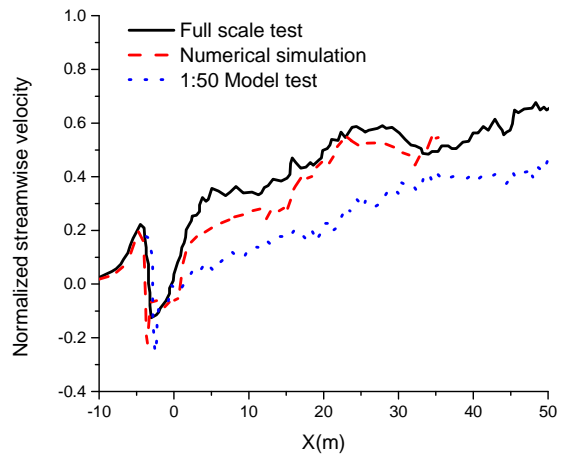

a

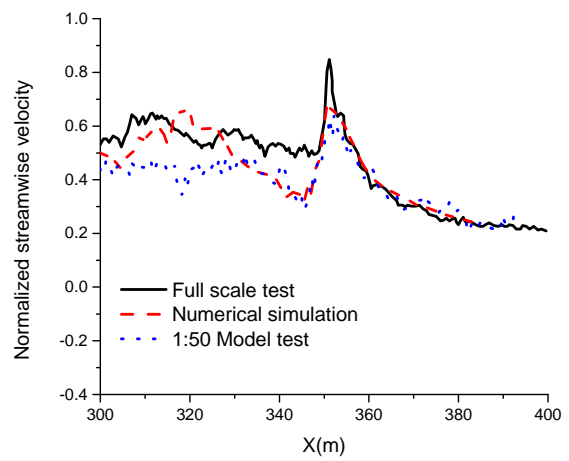

$b$

Fig. 6. Comparison in the normalized mean velocity component at the height of top rail obtained using full-scale, model scale tests and numerical simulation: (a) head; (b) tail.

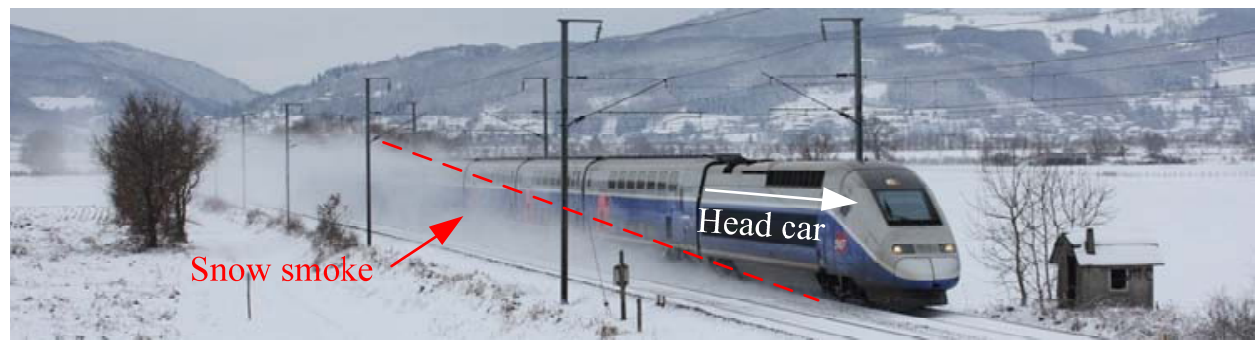

a

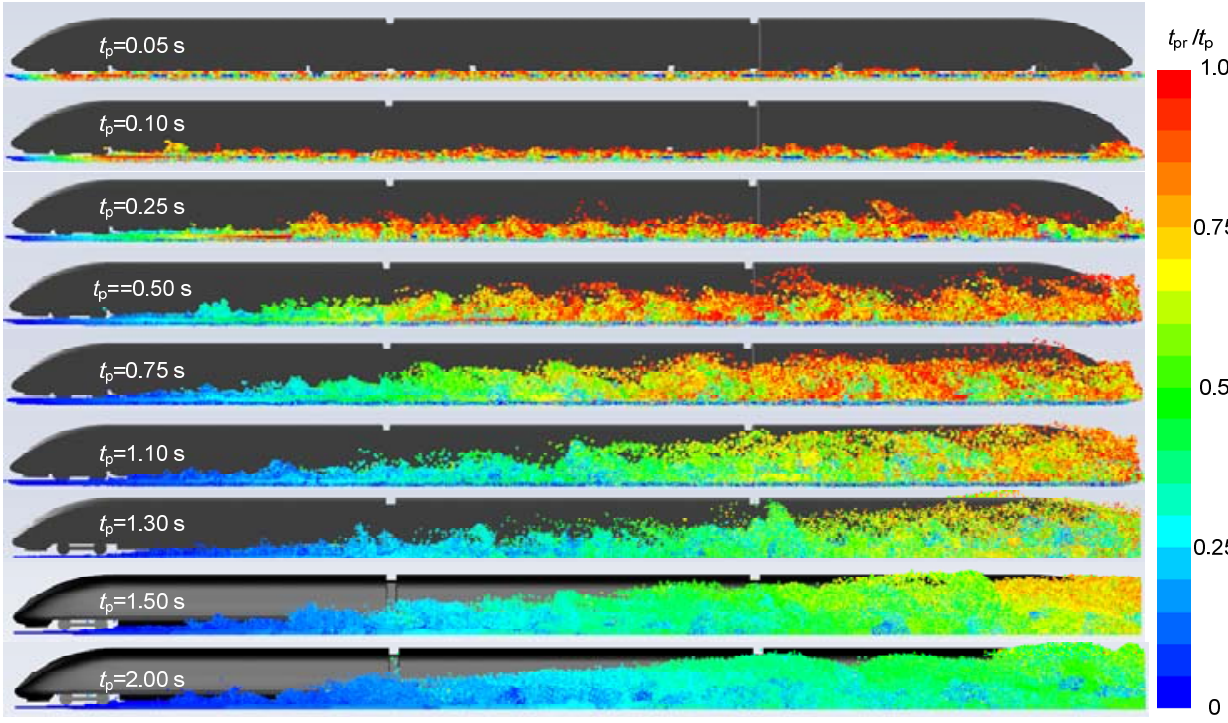

b

Fig. 7. Snow particles distribution around the train: (a) snow smoke around train (Paradot et al. 2014); (b) snow particles distribution around the train at different times.

the snow concentration on the side of the skirt plate is about twice as small as that underneath the car.

\subsection{Effects of Anti-Snow Deflector on Snow Accretion of Bogie}

Table 2 shows the change rates of snow particles captured by each car body and bogies for different cases during $t_{\mathrm{p}}=1.0 \sim 2.0 \mathrm{~s}$. From the comparison between Case 2 and Case 1, it can be seen that when the deflector is installed at the bogie 2 , the snow particles captured by the bogie 2 are reduced by about $59.6 \%$ for Case 2 . However, the snow 
L. Cai et al. / JAFM, Vol. 14, No. 1, pp. 287-299, 2021.

Table 2 Change rates of snow particles captured on each part in different case within $1.0 \mathrm{~s}$ to $2.0 \mathrm{~s}$

\begin{tabular}{|c|c|c|c|c|c|}
\hline \multirow{2}{*}{ Parts } & \multicolumn{2}{|c|}{ Total number of captured snow particles } & \multicolumn{2}{c|}{ Change rates } \\
\cline { 2 - 6 } & Case 1 & Case 2 & Case 3 & Case 2 & Case 3 \\
\hline Bogie 1 & 1665 & 2367 & 1014 & 0.422 & -0.391 \\
\hline Bogie 1 cavity & 2341 & 2167 & 1774 & -0.074 & -0.242 \\
\hline Bogie 2 & 754988 & 304474 & 770589 & -0.597 & 0.021 \\
\hline Bogie 2 cavity & 252036 & 276774 & 283001 & 0.098 & 0.123 \\
\hline Bogie 3 & 1194021 & 721065 & 1454058 & -0.396 & 0.218 \\
\hline Bogie 3 cavity & 461380 & 480326 & 559440 & 0.041 & 0.213 \\
\hline Bogie 4 & 1382581 & 987232 & 838483 & -0.286 & -0.394 \\
\hline Bogie 4 cavity & 548800 & 424002 & 679404 & -0.227 & 0.238 \\
\hline Bogie 5 & 1443515 & 1125120 & 1216863 & -0.221 & -0.157 \\
\hline Bogie 5 cavity & 724119 & 484889 & 691298 & -0.330 & -0.045 \\
\hline Bogie-6 & 1469200 & 923798 & 1072566 & -0.371 & -0.270 \\
\hline Bogie 6 cavity & 695122 & 411812 & 501919 & -0.408 & -0.278 \\
\hline Carbody head & 594328 & 727594 & 616137 & 0.224 & 0.037 \\
\hline Carbody mid & 2382022 & 3083932 & 3185833 & 0.295 & 0.337 \\
\hline Carbody tail & 3202513 & 2370367 & 3592394 & -0.260 & 0.122 \\
\hline Deflector & - & 340514 & 486162 & - & - \\
\hline
\end{tabular}

particles captured on the inner surface of the bogie 2 cavity are increased by $9.8 \%$. The total number of snow particles captured on the walls of the entire region of bogie 2 (bogie 2 and bogie 2 cavity) for Case 2 is reduced by $42.3 \%$. Meanwhile, due to the impact of the upstream deflector installed in the front end of the bogie 2, the snow particles captured by bogies $3,4,5$ and 6 are reduced by $39 \%, 28 \%$, $22 \%$ and $37 \%$, respectively. Moreover, it results in the number of snow particles captured by the head and middle car increased by $22 \%$ and $29 \%$, respectively. Although the deflector at the bogie 2 can reduce the number of snow particles captured on the walls which is adjacent to the bogie 2. It will disturb more snow particles from ground, resulting in more snow particles captured by the downstream car body.

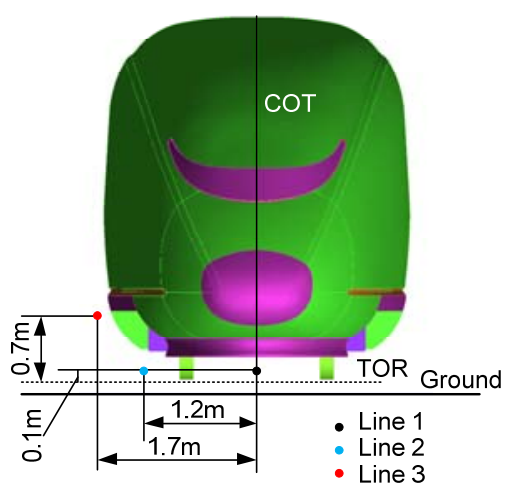

Fig. 8. Locations of monitoring lines of snow particle concentration.

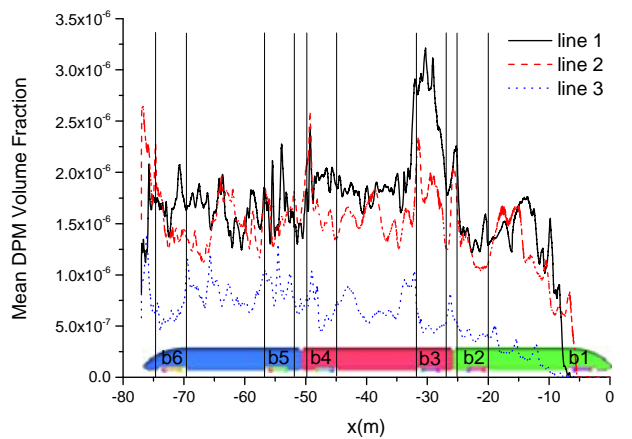

Fig. 9. Mean volume fraction of snow particle at the monitoring line for Case 1.

From the comparison between Case 3 and Case 1, it shows that when the deflector is installed at the bogie 4 , the snow particles captured by the bogie 4 are reduced by about $39.4 \%$. However, the snow particles captured on the inner surface of the bogie 4 cavity are increased by about $23.8 \%$. Although the deflector can significantly reduce the number of snow particles hitting the bogie 4, it will also significantly increase the number of snow particles hitting the walls of the bogie 4 cavity. The amount of snow particles captured on the bogie 4 region (bogie 4 and bogie 4 cavity) is reduced by only about $15.6 \%$. In addition, due to the impact of the deflectors at the bogie 4 , the number of snow particles captured by the middle and the tail cars increase by $33.7 \%$ and $12.2 \%$, respectively. This features show that the deflector at the bogie 4 can reduce the number of snow particles captured on the walls adjacent to the bogie 4. Meanwhile, it will also disturb more snow particles, resulting in more 

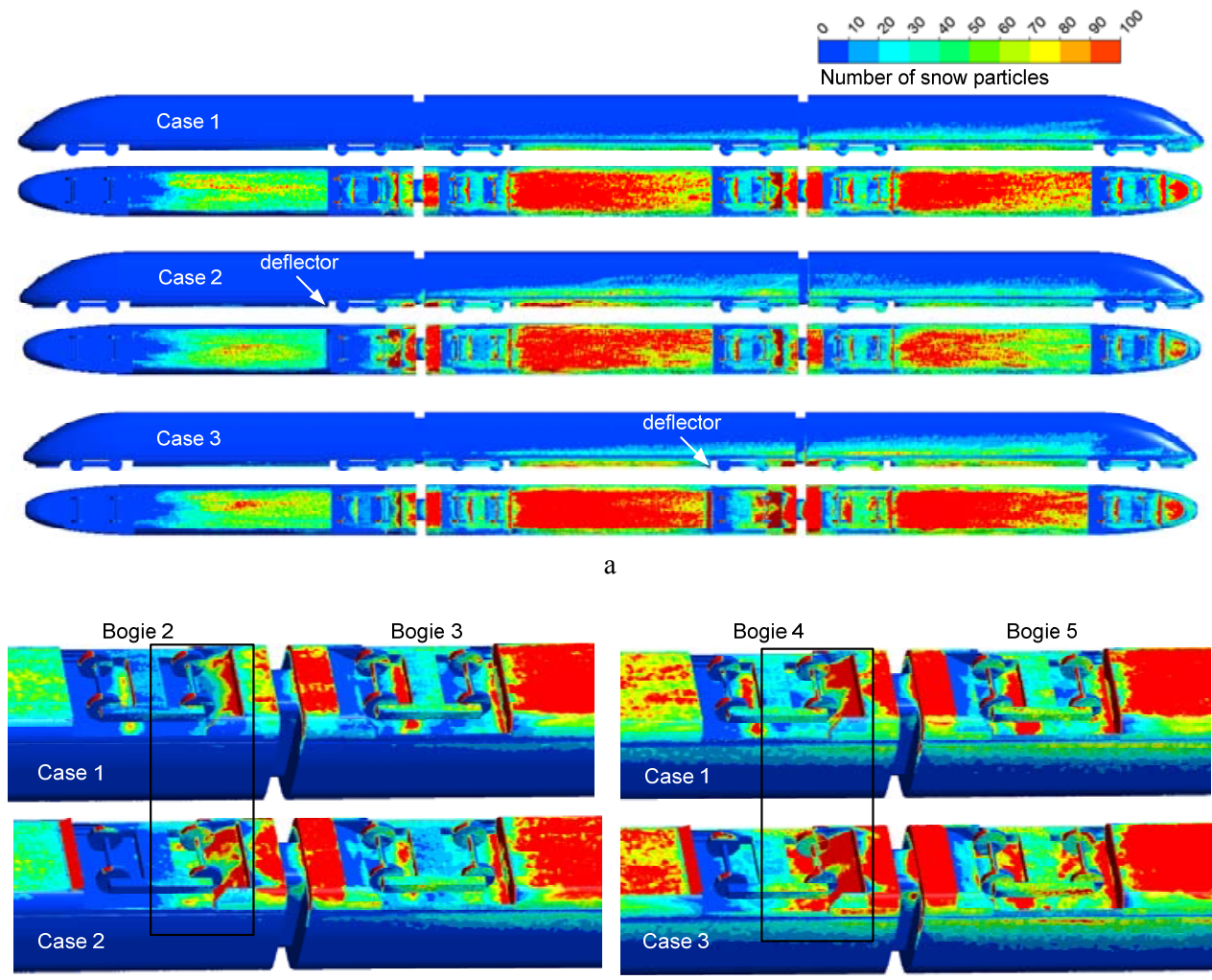

b

Fig. 10. The number of snow particles captured on the wall within 0 to $2.0 \mathrm{~s}$ : (a) the total number of snow particles captured on the wall within 0 to $2.0 \mathrm{~s}$; (b) comparison in the total number of snow particles captured on the wall of the bogie region among Case 1, Case 2 and Case 3.

snow particles captured by the downstream car body.

Figure 10 presents the distribution of the number of snow particles captured by the train body and the bogie walls during $t_{\mathrm{p}}=2.0 \mathrm{~s}$. By comparing Case 2 with Case 1, it can be seen that the deflector effectively reduces the number of snow particles captured in the front part of the bogie 2. However, it also increases the number of snow particles captured in the rear part of the bogie 2. At the same time, the deflector at the bogie 2 will increase the number of snow particles captured on the side of the middle car. And the height of the capture area of the snow particle is also higher. It is noted that the number of snow particles captured on the region of the bogie 6 and the bottom region of the tail car is reduced. The reason may be that the deflector of the head car causes the middle car to capture more snow particles, resulting in lesser snow particles being captured by the tail car.

With comparison to Case 1, the deflector for Case 3 can effectively reduce the number of snow particles captured in the front part of the bogie 4. But also significantly increase the number of snow particles captured by the bogie 4 cavity and car body at the rear of the bogie 4 . In addition, the deflector at the bogie 4 of middle car will increase the number of snow particles captured on the side of the downstream tail car, and the height of the capture area of the snow particle is also higher.

In general, the deflector can significantly reduce the number of snow particles hitting the wall of the front part of the bogie. It slightly increases the number of snow particles hitting the wall of the rear region of the bogie. When the deflector is installed at the bogie 2 or 4 , the snow particles captured in the bogie region are reduced by $42.3 \%$ and $15.6 \%$, respectively. The effect of preventing snow in the bogie region is different with the deflector in a different bogie position. The anti-snow performance of the deflector at the front of the train is better than that in the rear.

Figure 11 shows the time-averaged volume fraction of snow particles along the longitudinal monitoring lines for the three cases. With comparison to Case 1 , it can be seen that the snow particle concentration at the bottom of bogie 2 (lines 1 and 2) decreases at first and then increase along the flow direction for Case 2. At the same time, the deflector can obviously reduce the snow concentration below the downstream middle car. Moreover, the influence range of the deflector extends to the whole underbody of middle car. The impact of the deflector of the bogie 2 on the snow concentration in the edge beneath the bogie (line 2) is less than that in line 1. Comparing Case 3 with Case 1, it 
shows that the impact of the deflector of the bogie 4 on the snow particle concentration on downstream car is similar to that of the deflector at the bogie 2 . However, the concentration variation downstream deflector is smaller than that for Case 2 .

With comparison to Case 1, due to the influence of the deflector of the bogie for both Cases 2 and 3, the snow particle concentration on the side of skirt plate (line 3 ) of the bogie 2 or 4 is significantly increased. Furthermore, the snow concentration on the side of the downstream skirt plate of the bogie 3 and 5 decreases at first and then increases. In Case 2 , the snow particle concentration on the side of the tail car skirt plate slightly decreased. Since the position of line 3 is higher than lines 1 and 2, the snow particle concentration at line 3 is much lower than that of lines 1 and 2 .
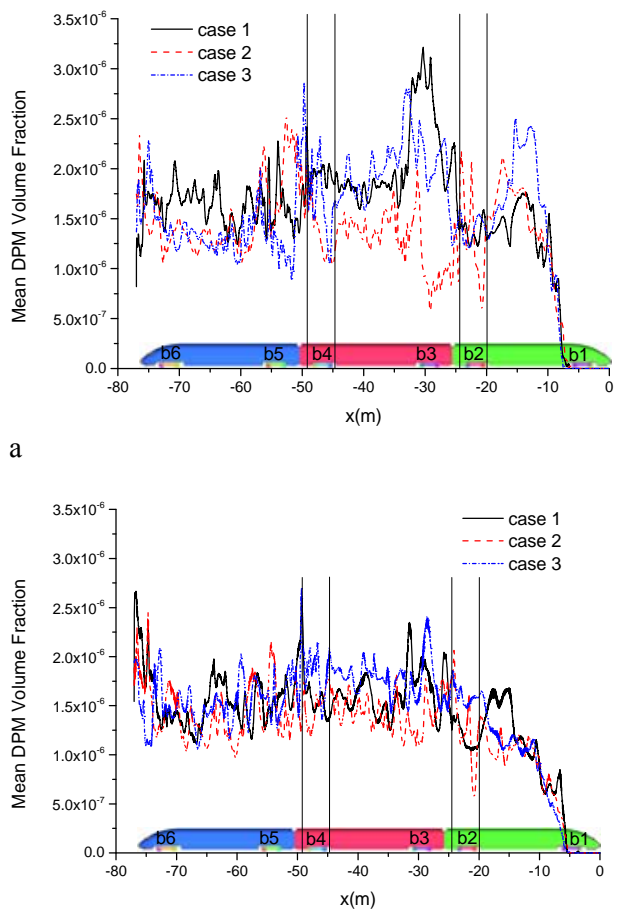

B

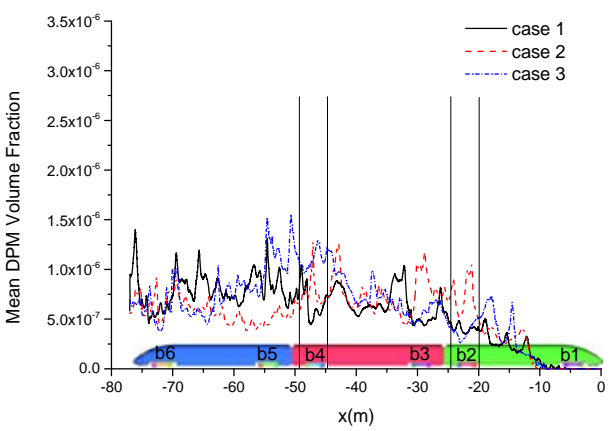

c

Fig.11. Time-averaged volume fraction of snow particles along longitudinal monitoring line within 2.0 s to 2.5 s: (a) line 1; (b) line 2; (c) line

3.
In general, the deflector can reduce the snow particle concentration beneath the car, but increase the snow particle concentration on the downstream skirt plate side. These areas may be more susceptible to the direct impact by the deflector and are more prone to accumulate snow.

Figure 12 shows the turbulent kinetic energy distribution at the ejection surface beneath the train for the three cases. For Case 2, the turbulent kinetic energy in the lower region of the bogie 2 and 3 was larger than that for Case 1. For Case 3, the turbulent kinetic energy in the lower region of the bogie 4 and 5 was also larger than that for Case 1. Due to the effects of the deflector installed in front of the bogies 2 or 4 cavities, the turbulent kinetic energy in the lower region of the bogie increases obviously. The high turbulence intensity helps the airflow to blow more snow particles on the ground and increases the amount of snow particles carried by the downstream airflow.

To further explain the impact of the deflector on snow transportation underneath the train, the vortex cores around the bogie for the three cases are show in Fig. 13. It is post-processed using commercial software EnSight 2019R1. The vortex cores help visualize the centers of swirling flow. Comparing with Case 1, more vortex cores are close to the ground below the bogie 2 or 4 downstream the deflector for Cases 2 or 3 . The vortex shedding at the edge of the bogie and the deflector will be guided toward to the ground, and the time and position of the downwash vortices reaching the ground will be advanced compared to the original models. Furthermore, the downstream vortices behind the deflector will suck up more snow particles on the ground, thereby causing the amount of snow particles captured on the downstream wall increased.

\section{CONCLUSION}

The influence of the anti-snow deflector on the wind-snow flow underneath the high-speed train is investigated using the numerical simulation combining IDDES approach and DPM. Based on the results of numerical simulation, the following conclusions can be drawn.

(1) The wind-snow flow around the train gradually develops from the head car to the tail car, increases gradually backward, and reaches the equilibrium state at the tail car. The concentration of snow particles beneath the train increases gradually from the head car, and gradually reaches a stable level at the central of the middle car.

(2) The deflector can significantly reduce the number of snow particles hitting the wall of the front region of the bogie. Meanwhile, the deflector can also disturb more snow particles from ground, resulting in more snow particles captured by the wall of the rear region of the bogie and the downstream car body. In addition, the deflector leads to an increase in the snow particle concentration on the side of the downstream skirt plate. 


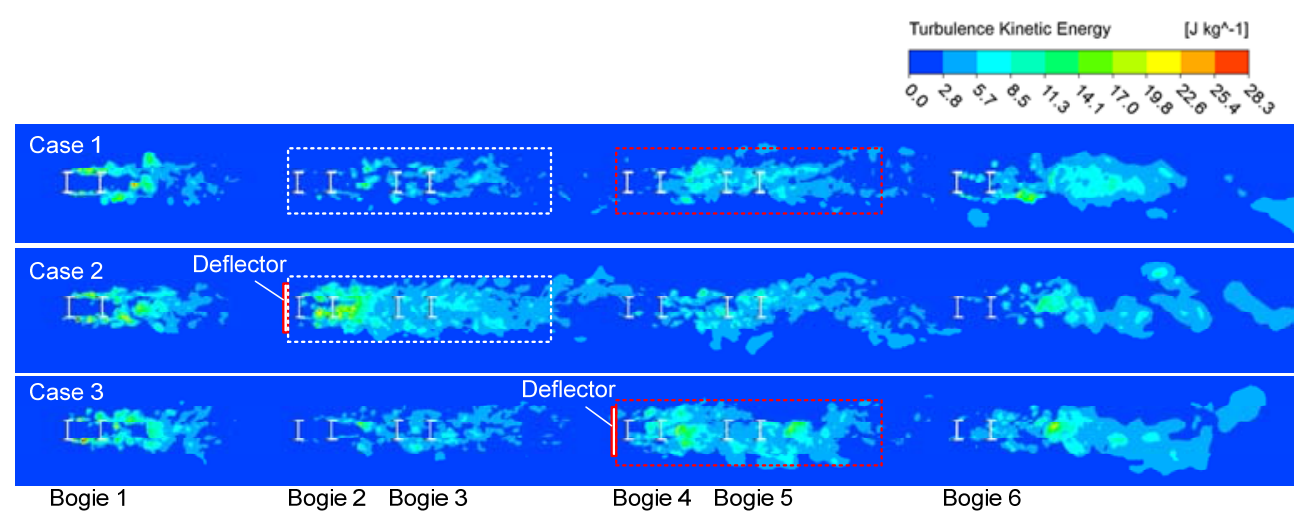

Fig. 12. Turbulent kinetic energy in the ejection surface at $t_{\mathrm{p}}=2.0 \mathrm{~s}$.

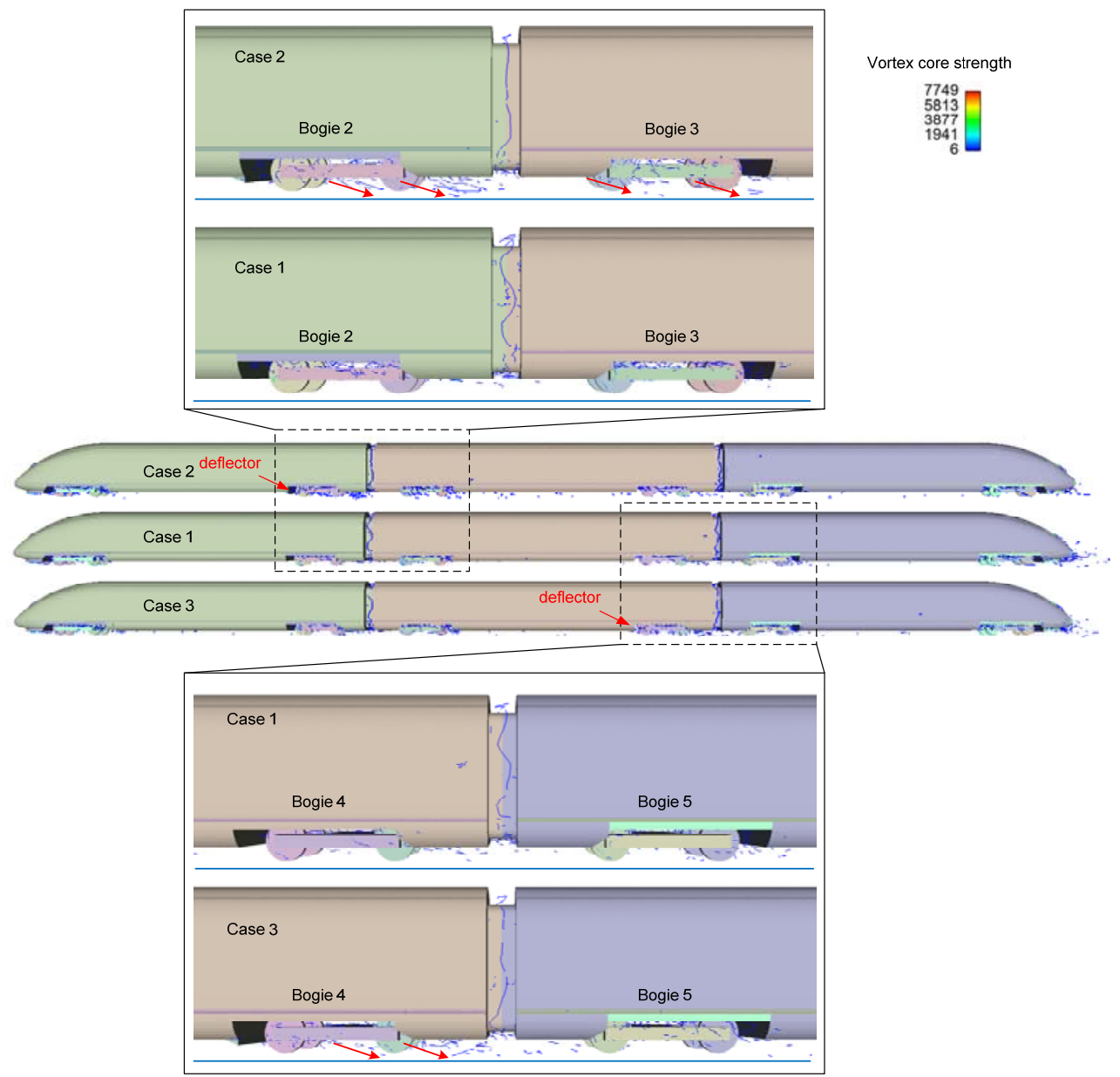

Fig. 13. Vortex core lines around the bogie at $t_{\mathrm{p}}=2.0 \mathrm{~s}$.

(3) The deflector guides the vortex shedding from the bogie to the ground, which increases the intensity of turbulent kinetic energy beneath the bogie. Furthermore, the downstream vortices behind of the deflector suck up more snow particles on the ground, which increase the number of snow particles carried by the downstream airflow and captured on the downstream surface of the train.

(4) The installation position for the deflector has a certain effect on the snow accretion in the bogie region. When the deflector is installed at the bogie 2 or 4 , the snow particles captured in the bogie region are reduced by $42.3 \%$ or $15.6 \%$, respectively. 
L. Cai et al. / JAFM, Vol. 14, No. 1, pp. 287-299, 2021.

\section{ACKNOWLEDGEMENTS}

This work was supported by the National Key Research and Development Program of China (Grant No. 2016YFB1200403), China Postdoctoral Science Foundation (No. 2019M663550) and Selfdetermined Project of State Key Laboratory of Traction Power (2019TPL_T02).

\section{REFERENCES}

Chiesa, M., V. Mathiesen, J. A. Melheim and B. Halvorsen (2005). Numerical simulation of particulate flow by the Eulerian-Lagrangian and the Eulerian-Eulerian approach with application to a fluidized bed. Computers \& Chemical Engineering 29(2), 291-304.

Deeg, P., M. Jönsson, H. J. Kaltenbach, M. Schober and M. Weise (2008). Crosscomparison of measurement techniques for the determination of train induced aerodynamic loads on the track bed. Proceedings of the $B B A A$ VI. Milano, Italy: 20-24.

Dong, Q. L., H. Y. Xu and Z. Y. Yem (2018). Numerical investigation of unsteady flow past rudimentary landing gear using DDES, LES and URANS. Engineering Applications of Computational Fluid Mechanics 12(1), 689710.

Gao, G. J., Y. Zhang, F. Xie, J. Zhang, K. He, J. B. Wang and Y. N. Zhang (2019). Numerical study on the anti-snow performance of deflectors in the bogie region of a high-speed train using the discrete phase model. Proceedings of the Institution of Mechanical Engineers Part F-Journal of Rail and Rapid Transit 233(2), 141-159.

Gosman, A. and E. Loannides (1983). Aspects of computer simulation of liquid-fueled combustors. Journal of Energy 7(6), 482-490.

Gritskevich, M. S., A. V. Garbaruk, J. Schütze and F. R. Menter (2012). Development of DDES and IDDES formulations for the $\mathrm{k}-\omega$ shear stress transport model. Flow, Turbulence and Combustion 88(3), 431-449.

Guilmineau, E., G. B. Deng and J. Wackers (2011). Numerical simulation with a DES approach for automotive flows. Journal of Fluids and Structures 27(5-6), 807-816.

Hedges, L. S., A. K. Travin and P. R. Spalart (2002). Detached-Eddy simulations over a simplified landing gear. Journal of Fluids Engineering-Transactions of the ASME 124(2), 413-423.

Jönsson, M., C. Wagner and S. Loose (2014). Particle image velocimetry of the underfloor flow for generic high-speed train models in a water towing tank. Proceedings of the Institution of Mechanical Engineers Part FJournal of Rail and Rapid Transit 228(2), 194209.
Kamata, Y. and A. Yokokura (2019). Estimation method of snow accretion amount on train bogies. Int. Workshop on Atmospheric Icing of Structures. Reykjavik.

Kang, S. O., S. O. Jun, H. I. Park, K. S. Song, J. D. Kee, K. H. Kim and D. H. Lee (2012). Actively translating a rear diffuser device for the aerodynamic drag reduction of a passenger car. International Journal of Automotive Technology 13(4), 583-592.

Kapadia, S., S. Roy, M. Vallero, K. Wurtzler and J. Forsythe (2003). Detached-Eddy simulation over a reference Ahmed car model. Proceedings of the fifth international ERCOFTAC Workshop on direct and largeeddy simulation, Munich University of Technology, Springer.

Kloow, L. (2011). High-speed train operation in winter climate. Stockholm.

Li, T., D. Qin and J. Y. Zhang (2019). Effect of RANS turbulence model on aerodynamic behavior of trains in crosswind. Chinese Journal of Mechanical Engineering 32(1), 112.

Li, T., Z. Y. Dai and W. H. Zhang (2020). Effect of RANS model on the aerodynamic characteristics of a train in crosswind using DDES. Computer Modeling in Engineering and Sciences 122(2), 555-570.

Milosevic, M. S., D. S. Stamenkovic, A. P. Milojevic and M. M. Tomic (2012). Modeling thermal effects in braking systems of railway cars. Thermal Science 16, S515-S526.

Nategh, S., H. Zhang, O. Wallmark, A. Boglietti, T. Nassen and M. Bazant (2019). Transient thermal modeling and analysis of railway traction motors. IEEE Transactions on Industrial Electronics 66(1), 79-89.

Pankajakshan, R., B. J. Mitchell and L. K. Taylor (2011). Simulation of unsteady two-phase flows using a parallel Eulerian-Lagrangian approach. Computers \& Fluids 41(1), 20-26.

Paradot, N., E. Allain, R. Croué, X. Delacasa and J. Pauline (2014). Development of a numerical modeling of snow accumulation on a high speed train. International Conference on Railway Technology: Research, Development and Maintenance. J. Pombo, Civil-Comp Press.

Patankar, N. A. and D. D. Joseph (2001). Modeling and numerical simulation of particulate flows by the Eulerian-Lagrangian approach. International Journal of Multiphase Flow 27(10), 1659-1684.

Paz, C., E. Suarez and C. Gil (2017). Numerical methodology for evaluating the effect of sleepers in the underbody flow of a high-speed train. Journal of Wind Engineering and Industrial Aerodynamics 167, 140-147.

Paz, C., E. Suarez, C. Gil and A. Cabarcos (2019). 
L. Cai et al. / JAFM, Vol. 14, No. 1, pp. 287-299, 2021.

Effect of realistic ballasted track in the underbody flow of a high-speed train via CFD simulations. Journal of Wind Engineering and Industrial Aerodynamics 184, 1-9.

Petry, M., A. Lamjahdy, A. Jawad, B. Markert and H. Murrenhoff (2018). Validation of a thermoand a hydromechanical model of a brake system for high-speed rail applications. Proceedings of the Institution of Mechanical Engineers Part F-Journal of Rail and Rapid Transit 232(8), 2149-2162.

Shishido, M., K. Nakade and A. Ido (2009). Development of deflector to decrease snowaccretion to truck of a car. Rtri Report 23(3), 29-34.

Shur, M. L., P. R. Spalart, M. K. Strelets and A. K. Travin (2008). A Hybrid RANS-LES approach with Delayed-DES and Wall-Modelled LES capabilities. International Journal of Heat and Fluid Flow 29(6), 1638-1649.

Sima, M., A. Gurr and A. Orellano (2008). Validation of CFD for the flow under a train with 1: 7 scale wind tunnel measurements. Proceedings of the BBAA VI International Colloquium on Bluff Bodies Aerodynamics and Applications. Milano, Italy.

Soper, D., D. Flynn, C. Baker, A. Jackson and H. Hemida (2018). A comparative study of methods to simulate aerodynamic flow beneath a high-speed train. Proceedings of the Institution of Mechanical Engineers Part FJournal of Rail and Rapid Transit 232(5), 1464-1482.

Sreenivas, K., R. Pankajakshan, B. Mitchell, L. Taylor and D. Whitfield (2006). Aerodynamic simulation of heavy trucks with rotating wheels. 44th AIAA Aerospace Sciences Meeting and Exhibit. Reno, AIAA. 22.

Tominaga, Y. (2018). Computational fluid dynamics simulation of snowdrift around buildings: Past achievements and future perspectives. Cold Regions Science and Technology 150, 2-14.

Vatsa, V. N. and D. P. Lockard (2010). Assessment of Hybrid RANS/LES turbulence models for aeroacoustics applications. 16th AIAA/CEAS Aeroacoustics Conference. Stockholm, Sweden, AIAA.

Wang, J. B., G. J. Gao, Y. Zhang, K. He and J. Zhang (2019a). Anti-snow performance of snow shields designed for brake calipers of a high-speed train. Proceedings of the Institution of Mechanical Engineers Part F-Journal of Rail and Rapid Transit 233(2), 121-140.

Wang, J. B., J. Zhang, F. Xie, Y. Zhang and G. J. Gao (2018a). A study of snow accumulating on the bogie and the effects of deflectors on the de-icing performance in the bogie region of a high-speed train. Cold Regions Science and
Technology 148, 121-130.

Wang, J. B., J. Zhang, Y. Zhang, F. Xie, S. Krajnovic and G. J. Gao (2018b). Impact of bogie cavity shapes and operational environment on snow accumulating on the bogies of high-speed trains. Journal of Wind Engineering and Industrial Aerodynamics 176, 211-224.

Wang, J. B., J. Zhang, Y. Zhang, X. F. Liang, S. Krajnovic and G. J. Gao (2019b). Impact of rotation of wheels and bogie cavity shapes on snow accumulating on the bogies of highspeed trains. Cold Regions Science and Technology 159, 58-70.

Wang, S. B., D. Burton, A. Herbst, J. Sheridan and M. C. Thompson (2018). The effect of bogies on high-speed train slipstream and wake. Journal of Fluids and Structures 83,471-489.

Wang, S. B., J. R. Bell, D. Burton, A. H. Herbst, J. Sheridan and M. C. Hompson (2017). The performance of different turbulence models (URANS, SAS and DES) for predicting highspeed train slipstream. Journal of Wind Engineering and Industrial Aerodynamics 165, 46-57.

Xia, C., H. Wang, X. Shan, Z. Yang and Q. Li (2017). Effects of ground configurations on the slipstream and near wake of a high-speed train. Journal of Wind Engineering and Industrial Aerodynamics 168, 177-189.

Xiao, Z. X., J. Liu, K. Y. Luo, J. B. Huang and S. $\mathrm{Fu}$ (2013). Investigation of flows around a rudimentary landing gear with advanced Detached-Eddy-Simulation approaches. AIAA Journal 51(1), 107-125.

Xie, F., J. Zhang, G. Gao, K. He, Y. Zhang and J. Wang (2017). Study of snow accumulation on a high-speed train's bogies based on the discrete phase model. Journal of Applied Fluid Mechanics 10 (6), 1729-1745.

Zhang, J., J. B. Wang, Q. X. Wang, X. H. Xiong, and G. J. Gao (2018). A study of the influence of bogie cut outs' angles on the aerodynamic performance of a high-speed train. Journal of Wind Engineering and Industrial Aerodynamics 175, 153-168.

Zhang, J., J. Li, H. Tian, G. Gao and J. Sheridan (2016). Impact of ground and wheel boundary conditions on numerical simulation of the high-speed train aerodynamic performance. Journal of Fluids and Structures 61, 249-261.

Zhu, J. Y. and Z. W. Hu (2017). Flow between the train underbody and trackbed around the bogie area and its impact on ballast flight. Journal of Wind Engineering and Industrial Aerodynamics 166, 20-28. 